\title{
Archeologia Globale ad Egnazia: nuove acquisizioni dalla città e dal territorio
}

\author{
Gianluca Mastrocinque \\ Università degli Studi di Bari, Dipartimento di Studi Umanistici, \\ email: gianluca.mastrocinque@uniba.it
}

\begin{abstract}
The data presented are the preliminary results of the interdisciplinary project FIRB named Landscape archaeology of the Adriatic "Puglia" in the Roman Age: innovative technologies for sustainable planning and knowledge of cultural identity, carried out since 2013 by the University of Molise (with a leading role), the University of Bari and the Politecnico of Bari. The project deals with the old landscape of Egnazia through a global approach, underlining the role of settlement dynamics, exploitation of natural resources, manufacturing activities and trading and cultural exchanges within the Mediterranean basin. For the first time this topographic research has allowed a systematic review of the territory through a complete survey of the surface, mostly unknown before. The results of the survey are connected with the information about the old town, largely explored thanks to the Project Egnazia: from Digging to Evaluation directed by Raffaella Cassano since 2001. The analysis of the data, referring both to the town and the territory, allows the identification of a strong settling structure, which is subjected to many transformations from the Bronze Age till Late Antiquity. Only a single feature is always visible during the whole ancient history of this place: the settlement was deeply affected by the viability and the distribution of the "lame" (landform smaller than a ravine), deep furrows in the natural stony bedrock used as water tanks and a link road between the hills and the Adriatic Sea.

Keywords: Landscape Archaeology, Egnazia, Systematic Survey, Cultural Heritage, Landscape

La città e il territorio di Egnazia costituiscono il contesto campione del Progetto FIRB Archeologia dei paesaggi della Puglia adriatica in età romana: tecnologie innovative per una pianificazione sostenibile e una fruizione identitaria, a cui partecipano, dal 2013, l'Università del Molise come capofila, l'Università degli Studi di Bari Aldo Moro e il Politecnico di Bari. Il programma rivolge un approccio globale al paesaggio antico di Egnazia, con attenzione alle dinamiche insediative, allo sfruttamento delle risorse naturali, alle attività produttive e agli scambi commerciali e culturali con il bacino del Mediterraneo.

Nell'ambito del programma di ricerca, il gruppo dell'Università del Molise, guidato da Marilena Cozzolino che cura anche il coordinamento generale, si occupa in particolare dell'indagine geofisica nell'area urbana e nel territorio; l'équipe dell'Università di Bari, coordinata da chi scrive, conduce lo studio del territorio e l'elaborazione di nuovi sistemi di comunicazione dei paesaggi pluristratificati, in sinergia con l'Ateneo del Molise per le ricostruzioni tridimensionali; il gruppo del Politecnico di Bari, coordinato da Giacomo Martines, svolge lo studio architettonico anche nell'ottica del restauro.

Dal punto di vista topografico, l'unità di ricerca dell'Università di Bari si concentra in modo specifico sul territorio - noto finora soprattutto sulla base di rinvenimenti sporadici - attraverso la ricognizione sistematica di superficie, che fino al 2014 ha coperto 13,5 kmq lper i rinvenimenti sporadici Mangiatordi \& Campese 2008, dove si accenna anche ai risultati di un preliminare intervento esplorativo limitato a 120 ettari, riproposti fedelmente in Mangiatordi \& Fioriello
\end{abstract}


2013-2014: 127-30, senza tenere conto delle acquisizioni intanto maturate attraverso la ricognizione sistematica). L'obiettivo è delimitare l'estensione dell'Ignatinus ager e focalizzarne le principali caratteristiche insediative, anche in rapporto con la città, sulla quale sono state invece raccolte negli ultimi anni consistenti acquisizioni, nell'ambito del Progetto Egnazia: dallo scavo alla valorizzazione, diretto dal 2001 da Raffaella Cassano (Cassano, 2008-2009; 2009a; 2011; Cassano et al, 2004; 2007; 2013).

La lettura congiunta dei dati provenienti dalla città e dal territorio permette di iniziare a delineare una fitta articolazione insediativa che, durante tutta la storia antica di questo comparto, è condizionata dalla viabilità e dalla distribuzione delle "lame", profondi solchi del banco roccioso naturale, usati come collettori d'acqua e come direttrici su cui orientare i collegamenti stradali tra le alture collinari interne e il mare Adriatico (fig. 1).

Per l'età del Bronzo il villaggio fortificato, documentato negli anni Sessanta del Novecento sull'acropoli di Egnazia (Biancofiore et al, 19941995), non costituisce più un'evidenza isolata, ma si trova in rapporto, forse come central place, con altri villaggi distribuiti nel territorio, soprattutto lungo le lame di maggiore portata. Nel settore sud-orientale del territorio di Egnazia, finora interessato in modo sistematico dal survey, indicatori significativi provengono soprattutto dalla lama San Domenico, sul cui versante occidentale è stato individuato un villaggio molto esteso Imq 9100 la sola concentrazione; mq 23000 lo spargimentol e fittamente occupato, come indica la rilevante concentrazione di frammenti di ceramica d'impasto e da fuoco, unita a numerose macine frammentarie in pietra lavica e ai conglomerati di argilla che rimandano in modo chiaro agli elevati delle capanne, oltre che ad una buona attestazione di industria litica. Un versante di questo nucleo insediativo è occupato da grotte naturali, componente geomorfologica distintiva di questo territorio, che per questo periodo rappresenta un altro specifico attrattore dell'insediamento umano, per la possibilità di abitare nelle cavità e di utilizzarle come deposito, oltre che come spazio sacro. Nel sistema di occupazione rientrano, oltre ai villaggi, nuclei demici minori collocati lungo le lame, come è emerso dai siti individuati a Lama D’Antico, Masseria Pignatta, Masseria Margagnano e Masseria Mingariello, nonché una concentrazione riferibile ad una capanna presso località La Forcatella, ad una distanza di $1 \mathrm{~km}$ rispetto al villaggio di masseria San Domenico.

Per il periodo che va dall'età del Ferro agli inizi dell'età ellenistica, quando Egnazia ricade lungo il confine tra Messapia e Peucezia, le evidenze diventano più rare sia nell'area che in seguito sarà occupata dalla città sia nel territorio. Nel primo caso la scarsità di documentazione sembra dipendere soprattutto dal fatto che le strutture della città di età romana imperiale e tardoantica obliterano l'insediamento messapico a nuclei sparsi di capanne, con necropoli contigua. Il centro messapico presenta comunque un buon livello di organizzazione e un'estensione significativa, pari a circa 14 ettari alla fine del IV sec. a.C., quando Egnazia si dota di una prima sistemazione urbana con la costruzione del circuito murario e con la individuazione di aree di utilizzo collettivo. Per quanto riguarda il territorio, invece, l'esiguità di documentazione, anche in contesti pluristratificati, pare rimandare ad una rarefazione insediativa.

Per il periodo messapico, i dati da Egnazia, anche se parziali, sembrano al momento non contrastare con la tendenza ad un sistema di insediamenti accentrati e strutturati disposti su distanze rilevanti, che la ricerca sta chiarendo in altri siti della Puglia meridionale, in particolare nel territorio di Oria e nel brindisino (Burgers, 1998; Semeraro, 2009a: 80-84; 2009b: 292-93)

A partire dalla seconda metà del II sec. a.C. un nuovo mutamento interessa la città, con ripercussioni anche nell'ager, entro un ampio periodo che arriva all'età augustea e alla prima età imperiale. All'interno delle mura è questa la fase in cui prende forma la maglia urbana a partire dalla rete stradale, secondo dinamiche che caratterizzano il paesaggio della città fino alla destrutturazione della fine del VI sec. d.C. Un ruolo prioritario è assegnato alla via 


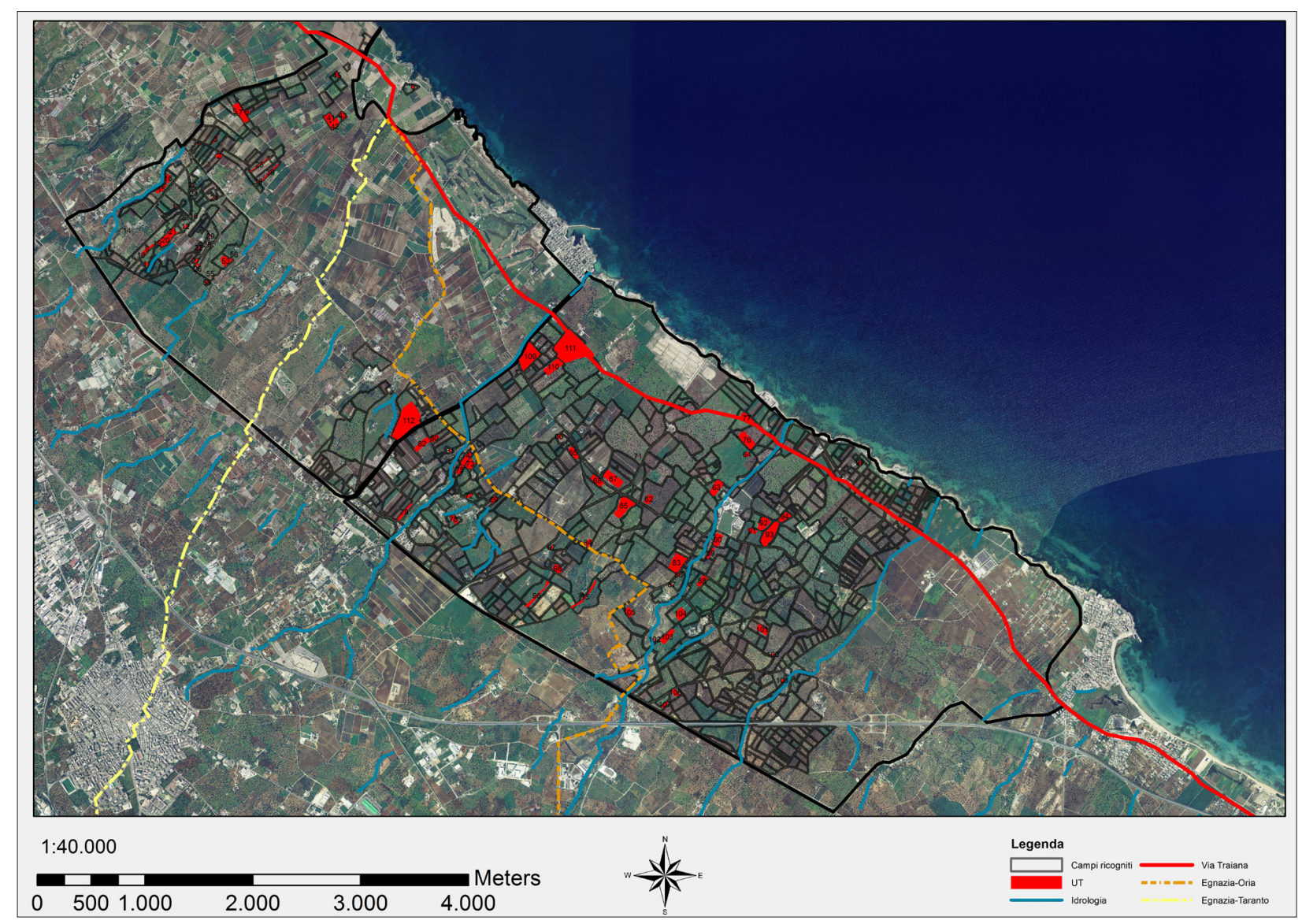

Fig. 1. Immagine satellitare di Egnazia. Carta generale con indicazione delle unità topografiche individuate in relazione alla rete idrografica e alla viabilità antica (di M Campese).

Minucia, che attraversa Egnazia con funzione di decumanus maximus in direzione Ovest-Est, separa le aree pubbliche, concentrate tra la strada e l'acropoli, dagli isolati dedicati agli spazi residenziali e produttivi e prosegue alla volta di Brundisium, divenendo un asse di primo rilievo anche nell'organizzazione del territorio. La maglia urbana così definita accoglie gli spazi caratteristici della civitas, dove si intensifica l'edilizia pubblica soprattutto negli anni finali della Repubblica e all'inizio del periodo augusteo (Cassano, 2009b: 50-54). A questo proposito, la concessione del patronatus a M. Vipsanio Agrippa, documentata su base epigrafica (CIL IX 262), rimanda evidentemente ad un programma di potenziamento urbano, seguito direttamente da Agrippa, per rendere Egnazia uno scalo strategico per la flotta, a sostegno del porto di Brindisi, negli anni conclusivi della guerra civile. Questo giustifica l'attenzione riservata al porto, ma anche la creazione del Foro con i suoi annessi monumentali, in particolare la basilica civile e, sul lato meridionale del comparto forense, le terme (Mastrocinque, 2008; 2014; Guida Egnazia, 2015).

La riorganizzazione in atto tra la fine del I sec. a.C. e il I sec. d.C., con l'ampliamento dell'abitato e il conseguente incremento della popolazione urbana, induce a riferire a questo periodo anche l'impostazione della rete di approvvigionamento idrico, che non utilizza un acquedotto, ma si avvale di cisterne e di pozzi che captano direttamente la falda o raccolgono l'acqua meteorica.

A supporto della rete idrica urbana, ai margini dell'abitato vengono mantenute libere ampie aree destinate al rifornimento. Lo scavo stratigrafico ha evidenziato uno di questi spazi nel settore Nord-orientale, subito all'interno delle mura, 
dove sono emersi pozzi di captazione e "polle" con risalita di acqua di falda. Ancora più estesa è l'area umida ipotizzabile sul lato nord ovest, all'esterno del circuito murario, utile soprattutto per le coltivazioni intensive che devono aver interessato la fascia suburbana, a giudicare dalla scarsezza di tracce di insediamento entro un raggio di 2,5 km circa. In questo caso l'assenza di evidenze archeologiche non impedisce di rintracciare alcuni "relitti" del paesaggio antico. In particolare, il consistente deposito limo-sabbioso, che interessa questo comparto in modo abbastanza uniforme, potrebbe essere l'esito del prosciugamento di zone umide antiche. Una conferma sembra venire dalla toponomastica che rimanda in modo quasi univoco alla presenza di aree libere: questo settore porta, infatti, ancora oggi il nome di “i Pantanelli" ed è seguito, a poca distanza a nord ovest, dalla zona chiamata in modo significativo “lumo", dal greco $\lambda i \mu v n$, specchio d'acqua ferma, ovvero dal latino limus, fango, che caratterizza in modo uniforme la matrice litologica di quest'area.

Lo studio del paleoambiente (Cuccovillo, 2010; Stellati, 2012-2013) segnala inoltre, specie per il V e il VI sec. d.C., un significativo ampliamento di aree umide di antica formazione e costante utilizzo: a questo proposito di particolare interesse sono le attestazioni faunistiche di specie come la gru (Grus grus), nonché i resti vegetali riferibili ad essenze tipiche di zone umide, in particolare la canna comune (Arundo donax) e il lino (Linum usitatissimum) che, seppur al momento documentato da un solo rinvenimento, riveste particolare importanza poiché si tratta di una specie difficilmente conservabile e in Puglia attestata solo ad Arpi.

A maggiore distanza dalla città, nel settore di territorio indagato si riscontra un netto aumento delle tracce di occupazione (fig. 2), segno di una ripresa dello sfruttamento, di cui il prosieguo del survey consentirà di tratteggiare in modo più preciso caratteristiche ed estensione.

Al periodo repubblicano è possibile riferire 34 Unità topografiche: sette sono riconducibili a tre distinte villae, altre dieci unità sono interpretabili come casa/fattoria, uno come sepolcreto e sedici sono gli spargimenti difficilmente riconducibili ad una precisa categoria insediativa. A questo riguardo è opportuno precisare che, per l'interpretazione delle UT, nel territorio di Egnazia trova sostanziale riscontro la tipologia elaborata da F. Cambi (2001) per la Puglia centrale e meridionale in età romana e medievale, basata su cinque criteri fondamentali lqualità, quantità e concentrazione dei reperti, dimensioni delle aree di spargimento e distribuzione dei reperti al loro interno), integrata con il repertorio formulato da M. Aprosio (2008) per il paesaggio brindisino.

La distribuzione dei siti evidenzia la predilezione per la vicinanza con le lame, oltre che con la viabilità secondaria e soprattutto con il percorso viario principale della via Minucia. In questo senso la situazione insediativa presenta specifiche affinità con il paesaggio di Brindisi, in particolare con il settore immediatamente all'interno rispetto alla costa, attraversato dalla via Appia, come emerge dalle analisi di dettaglio più recenti (Aprosio, 2008: in particolare 118-20).

Nell'ager di Egnazia, la documentazione più cospicua proviene dal settore che si trova subito all'interno della frazione di Savelletri, attraversato dalla Lama D'Antico - toponimo che sembra serbare memoria della lunga frequentazione antropica - e da un asse viario secondario, ipotizzabile sulla base della distribuzione delle evidenze e conforme nell'orientamento dall'interno verso il mare. Tra la strada e il solco della lama si dispongono, quasi in allineamento, un sito di particolare consistenza llocalità Masseria Burgol, con ogni probabilità già in questo periodo occupato da una villa che aggrega nelle vicinanze diverse unità abitative e, in sequenza, altri nuclei demici di rilevante entità (nelle località Masseria Calderisi e Masseria San Leonardo). La documentazione è rappresentata da ceramica di produzione e soprattutto di importazione, anfore italiche e africane che rimandano in particolare alla circolazione del vino e ceramica fine da mensa a pasta grigia, a pareti sottili e in terra sigillata, soprattutto italica.

Insieme all'impianto produttivo rurale documentato stratigraficamente a poca 


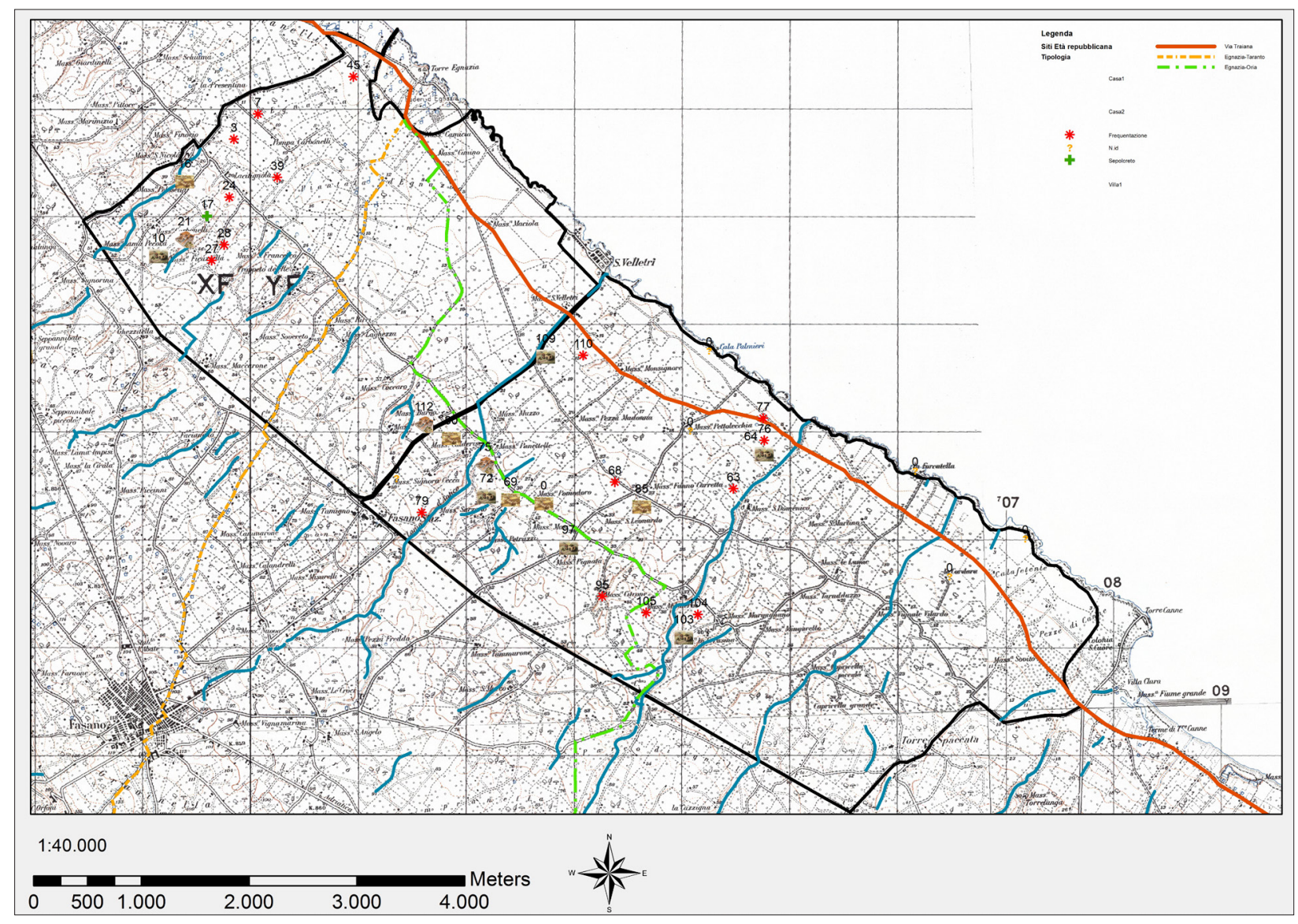

Fig. 2. Carta generale di Egnazia con indicazione dei siti di età repubblicana (di M Campese).

distanza, in località Vuotano Piccolo, attivo in età tardorepubblicana per la produzione di materiale fittile, soprattutto ceramica d'uso comune e fuseruole di cui restano scarti in ingente quantità (Cocchiaro, 1991: 275-76), questi rinvenimenti forniscono le prime evidenze del consolidarsi di interessi fondiari per lo sfruttamento agricolo intensivo e per l'allevamento, che in parte possono essere riferiti anche a famiglie non locali. Significativo in proposito è il bollo su tegola CETHEGI ET SCIPIONIS (CIL IX 6078, 58 tegulae), rinvenuto in questo stesso comparto nel XIX secolo - nell'area genericamente indicata come Masseria Pettolecchia - e di recente riferito da M. Silvestrini ad una proprietà, forse connessa ad un impianto produttivo, della famiglia dei Cornelii
Lentuli, ben documentata anche a Brindisi e ad Ausculum (Silvestrini, 2005: 116, nota 13). Nello stesso senso vanno attestazioni che riguardano le famiglie più in vista nella contesa politica e che spiegano con maggiore chiarezza il ruolo prioritario di questo distretto nello scacchiere militare della fine della Repubblica. Oltre a non poter escludere possedimenti diretti della famiglia del patronus Agrippa, infatti, si può anche pensare a fundi di proprietà della famiglia di Marco Antonio, dal momento che suo figlio Marco lullo Antonio è con ogni probabilità il patrono del liberto Marcus Antonius Sotericus (Chelotti, 2007: 479-80, n. 51), sepolto nella necropoli situata subito all'esterno delle mura, sul lato meridionale.

Al periodo imperiale è possibile riferire 19 Unità topografiche (fig. 3): sette sono riconducibili a tre distinte villae, altre sei unità sono interpretabili come casa/fattoria, una come sepolcreto e cinque sono gli spargimenti difficilmente riconducibili ad una precisa categoria insediativa. 


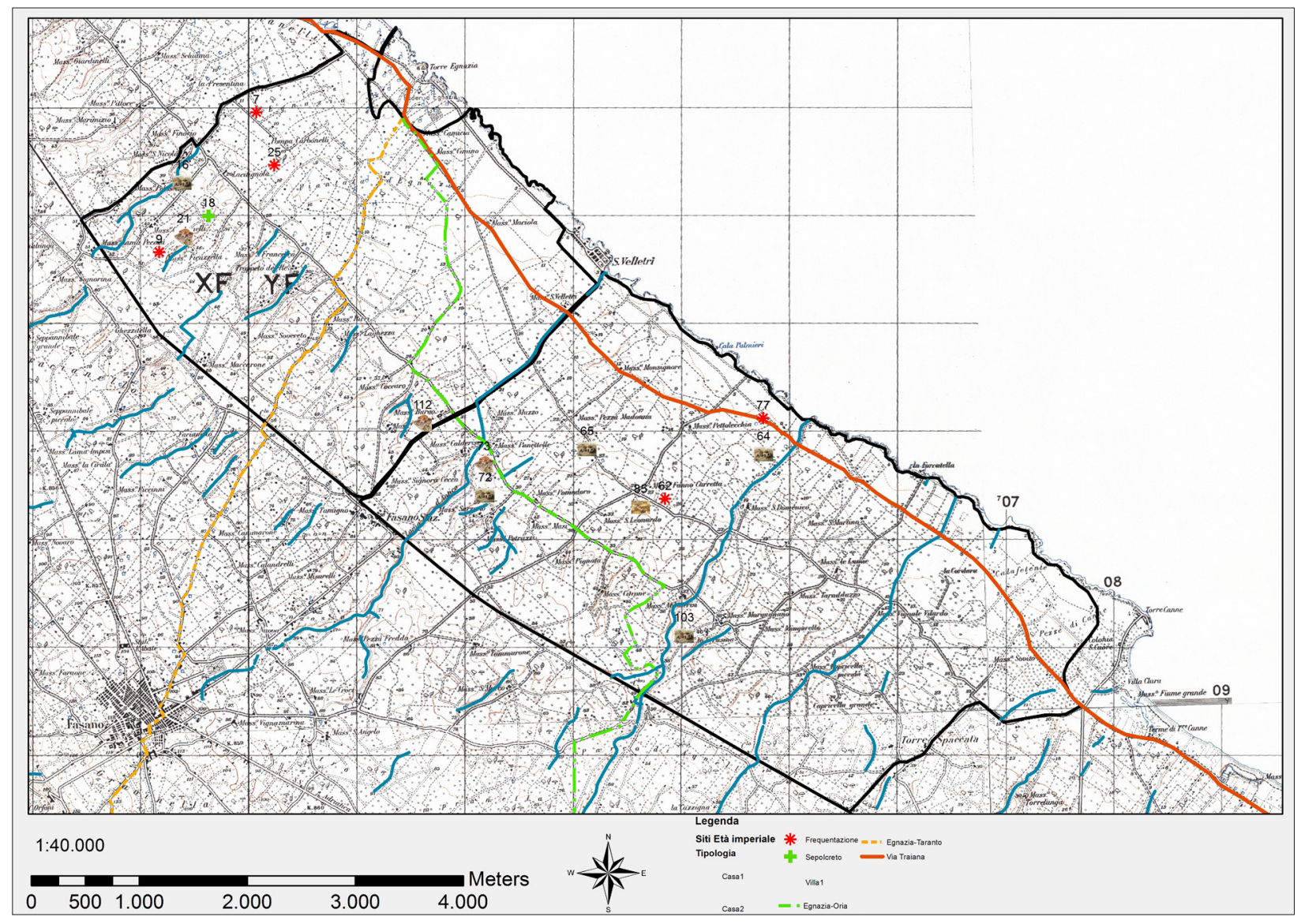

Fig. 3. Carta generale di Egnazia con indicazione dei siti di età imperiale (di M Campese).

Il calo numerico delle unità riferibili a questo periodo riguarda quasi esclusivamente le aree di spargimento non interessate da insediamenti strutturati, mentre si nota solo una lieve contrazione della casa/fattoria e una sostanziale persistenza degli insediamenti principali come le ville. Una chiara continuità si riscontra inoltre nel Il sec. d.C., quando il sistema viario è interessato da un potenziamento legato alla costruzione della via Traiana, in gran parte sul percorso della più antica via Minucia. Gli effetti di questo intervento sono al momento più evidenti dentro la città, dove l'invasivo cantiere per la messa in opera della strada favorisce un più esteso rimodellamento, che si adegua alla forma urbis già impostata nel secolo precedente, ristruttura diversi settori e si concentra soprattutto nelle zone più vicine alla via, anche con interventi architettonici di alto tenore monumentale (Cassano, 2009b: 55-60; Cassano et al; 2007: in particolare 17-31, 53-59, 69-74).

Nel settore più vicino alla costa, la documentazione, anche se non cospicua, per il periodo tra I e III secolo rimanda ad alcune abitazioni di ridotta estensione, tra cui spicca un sito prossimo alla foce di Lama San Domenico che ha restituito, oltre a laterizi, significativa concentrazione di ceramica d'uso comune e da fuoco, ceramica da cucina africana (forma Hayes 23B), anfore di produzione africana e adriatica, in particolare l'anfora vinaria Dressel 2-4, che in Puglia è documentata solo in questo ambito sub-regionale, a Seppannibale (D'Eredità \& Caringella, 2011: 459, tav. II, n. 4.1) e a Mola Paduano (Casavola, 2002: 77-80). Di notevole interesse è inoltre la concentrazione di indicatori di attività legate alla pesca, soprattutto 16 pesi da rete, presenza del tutto rilevante in termini 


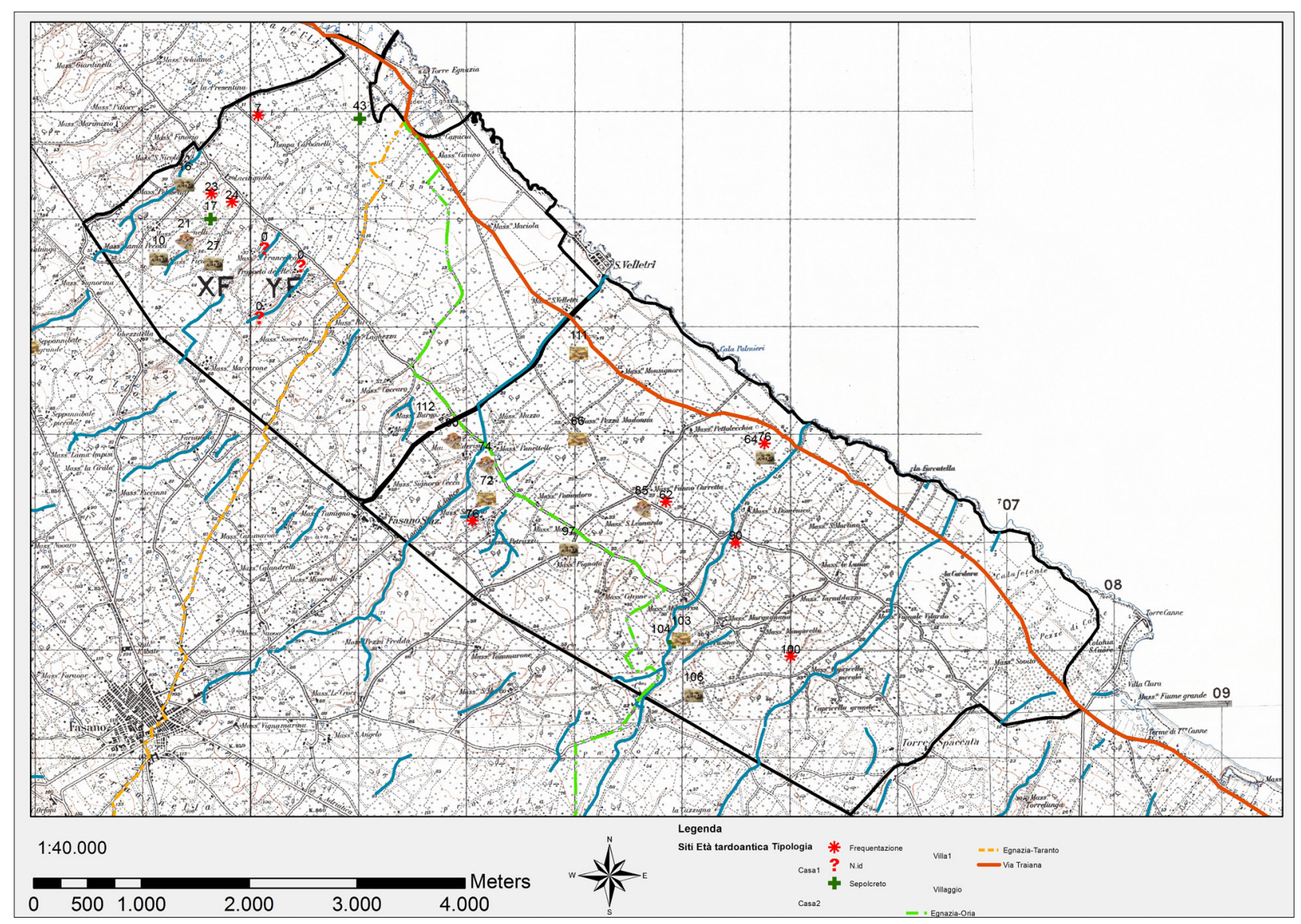

Fig. 4. Carta generale di Egnazia con indicazione dei siti di età tardoantica (di M Campese).

quantitativi in relazione all'estensione dell'unità topografica pari a 1500 mq. I materiali provenienti da questo contesto sembrano dunque fornire una sintesi efficace delle attività che completano il quadro economico del periodo imperiale, insieme all'agricoltura intensiva e all'allevamento.

Sul finire del IV secolo, la ricerca degli ultimi anni ha ormai chiarito un marcato mutamento insediativo nella città, a cui possono ora aggiungersi dati interessanti sul territorio. In seguito ad estese destrutturazioni, legate forse anche ad un evento calamitoso, come il sisma del 365 d.C. avvertito in varie località del Mediterraneo orientale, nel volgere di pochi decenni, tra la fine del IV e gli inizi del V secolo, il paesaggio urbano cambia volto (Cassano, 2007; 2008-2009; Cassano et al, 2013). Rispetto al precedente modello insediativo prevalgono due tendenze fondamentali: da un lato si affermano in modo netto le istanze aggregative e rappresentative del culto cristiano - espresse in particolare dalla concentrazione di edifici di culto in un "polo religioso" al centro della città -, dall'altro diventano predominanti le attività artigianali e commerciali, tanto che alcuni dei principali monumenti pubblici sono riconvertiti ad attività di produzione e di distribuzione su larga scala (Mastrocinque, 2014). È il caso della piazza del mercato, che accoglie magazzini per lo stoccaggio e per la vendita nello spazio riconvertito del quadriportico oppure del santuario dell'acropoli, ormai privo della funzione religiosa, dove si impianta un articolato complesso a carattere residenziale e produttivo, tra cui spicca una residenza organizzata intorno ad un atrio centrale, riferibile forse ad un alto esponente della guarnigione stanziata nella fortificazione bizantina della seconda metà del VI secolo. Nelle terme, invece, l'ingente dotazione dei marmi 

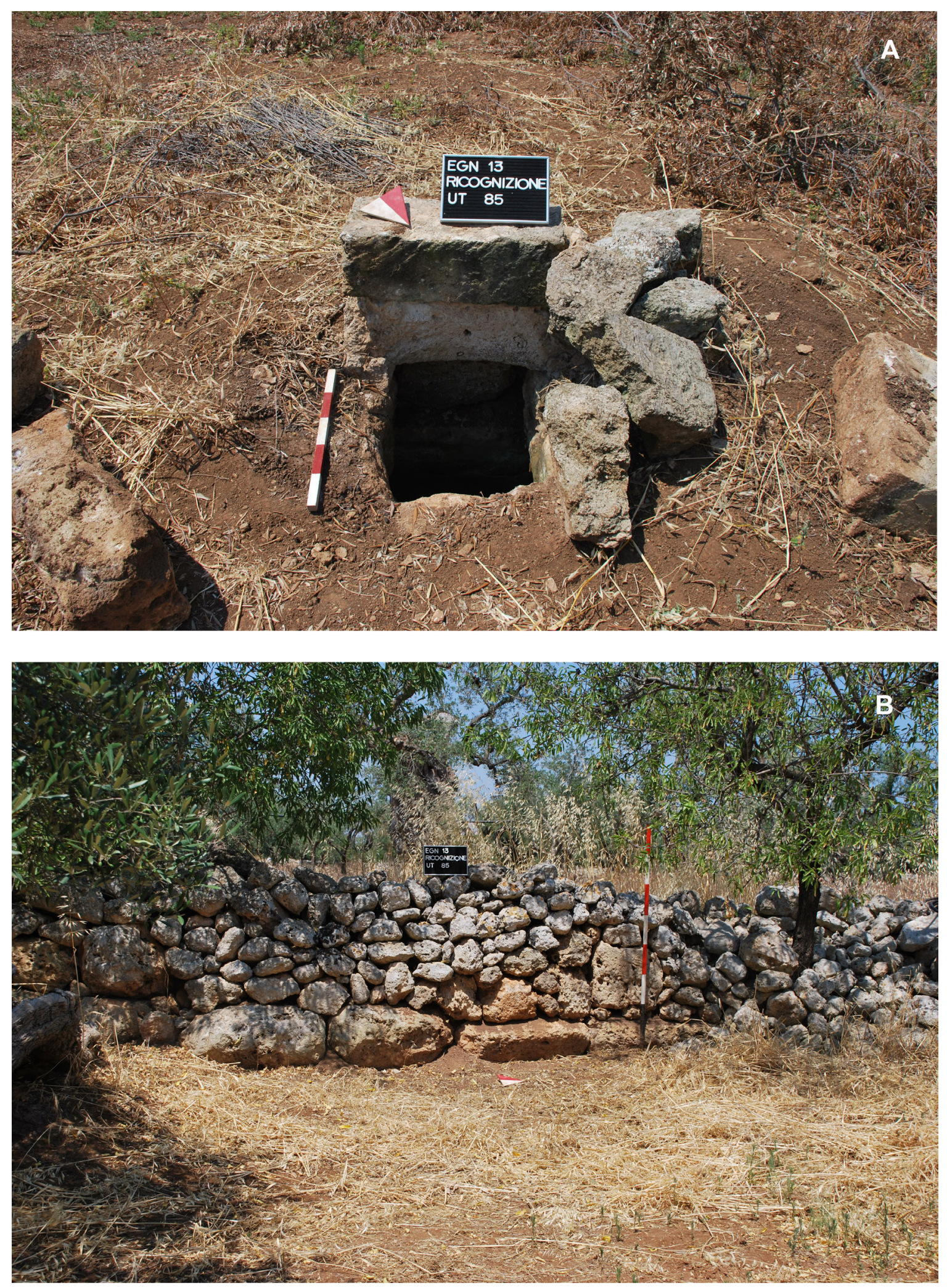

Fig. 5. Masseria San Leonardo, villa di età tardoantica: imboccatura della cisterna al e struttura in opera quadrata b), conservata per $271 \mathrm{~m}$ e riutilizzata come fondazione di muri interpoderali (foto: author). 
utilizzati nei rivestimenti favorisce l'impianto di un'articolata manifattura per la produzione di calce e di laterizi, richiesti dalla intensa attività edilizia della città Mastrocinque c.s.. A questi spazi artigianali si affiancano gli edifici privati in cui coincidono gli spazi dell'abitare e del produrre per l'autoconsumo, nonché talvolta anche aree per lo stazionamento e per l'allevamento del bestiame, secondo il sistema insediativo polifunzionale di cui la ricerca recente sta chiarendo un'ampia area di diffusione su scala mediterranea. Per restare in ambito pugliese e lucano, i confronti più stringenti, anche per l'articolazione degli spazi, riguardano Ordona, Venosa Giuliani, 2010: 13135) e il sito di Vagnari (Favia \& Giuliani, 2011: 184, 221-24; Small, 2011: 29-33), tutti contesti in cui la riorganizzazione insediativa prende avvio, come ad Egnazia, dalla seconda metà del IV secolo.

Gli aspetti principali del cambiamento evidenziano in modo chiaro non solo l'esito delle trasformazioni socio-economiche indotte dalla riforma di Costantino, ma soprattutto il nuovo ruolo assunto dal vescovo, che fonde l'autorità religiosa, rafforzata dalla maggiore diffusione del cristianesimo, con la gestione diretta di competenze amministrative e in particolare di attività economiche, in sostituzione delle magistrature municipali.

Il passaggio da una città “di consumo" ad una "città-società" a forte vocazione produttiva implica la riorganizzazione anche delle campagne: in particolare si nota un deciso ampliamento dei nuclei insediativi già attivi e la diversificazione delle attività in essi svolte, a cui rimanda una maggiore articolazione delle attestazioni della cultura materiale, soprattutto della ceramica. La distribuzione continua a prediligere i pianori situati oltre i $3 \mathrm{~km}$ dalla città, in stretta relazione con la viabilità. Una posizione che risulta strategica perché interessa la fascia intermedia tra la zona costiera, caratterizzata soprattutto dalle coltivazioni e dalle attività legate alla pesca, e i rilievi collinari verosimilmente interessati da spazi per l'allevamento e da zone boschive, ancora oggi in parte conservate nell'area che porta il significativo toponimo de "La Selva" di Fasano.
Al periodo tardoantico è possibile riferire 28 Unità topografiche, otto delle quali di nuovo impianto (fig. 4): otto sono riconducibili a quattro distinte villae, altre unità rimandano ad una villaggio, nove sono interpretabili come casa/ fattoria, due sono sepolcreti e otto sono gli spargimenti difficilmente riconducibili ad una precisa categoria insediativa.

La villa sembra l'esito del potenziamento dei nuclei già esistenti nei pressi di Masseria Calderisi e di Masseria San Leonardo, a cui possono aggiungersi analoghi insediamenti, di nuova impostazione, presso le attuali Masseria Carbonelli, disposti sempre a breve distanza dai solchi delle lame. L'evidenza archeologica presenta elementi coerenti in tutti i contesti: importanti concentrazioni di frammenti laterizi e ceramici, estese su aree comprese tra i 10.000 e i $15.000 \mathrm{mq}$, che documentano una frequentazione ininterrotta tra il IV e il VII secolo. Alla alta densità corrisponde una rilevante varietà delle classi ceramiche: vasellame fine da mensa di produzione africana e focese, contenitori da trasporto di produzione africana e orientale, nonché monete in bronzo ascrivibili al IV secolo e, nel sito di Masseria Calderisi, anche reperti vitrei e tessere di mosaico, che rimandano al nucleo residenziale dell'insediamento. Pienamente conservate risultano inoltre, nelle ville di Masseria Calderisi e Masseria San Leonardo, cisterne a sezione campaniforme, rimaste in uso in alcuni casi fino al secolo scorso e confrontabili con analoghi dispositivi della città di questo periodo, come nel settore a sud del Foro, nell'area aperta prossima all'edificio religioso a pianta longitudinale di recente individuato, che oblitera una domus ad atrio di notevole prestigio. Particolarmente significativa a questo riguardo è la cisterna pertinente alla villa di Masseria San Leonardo (fig. 5a), alla cui pars fructuaria rimanda anche una struttura muraria in opera quadrata ben commessa, di cui affiora un filare, e solo per esigui tratti un secondo filare, per una lunghezza complessiva di $\mathrm{m} 271$, riutilizzata come fondazione di muretti a secco interpoderali di epoca moderna, segno tangibile del palinsesto pluristratificato in questo territorio (fig. 5b). 
La densità di occupazione e lo sfruttamento intenso del settore di ager indagato emerge anche nel sito di Masseria Burgo dove la villa già attiva diviene un agglomerato demico di maggiore consistenza, interpretabile con buoni elementi come villaggio (fig. 4). L'insediamento è servito dal percorso viario già segnalato che conduceva ad un approdo nei pressi di Savelletri, con funzione complementare rispetto al porto della città, ancora in piena attività. Su un'estensione pari a circa $30.000 \mathrm{mq}$ è stata individuata una concentrazione del tutto rilevante di materiale ceramico, con una netta preponderanza di contenitori da trasporto: le anfore, pari all' $80 \%$ delle classi attestate, si riferiscono forse ad un settore dell'insediamento dedicato a spazi di stoccaggio. Nell'ambito del repertorio delle anfore, l'88\% proviene dall'Africa, con una predilezione netta nel $V$ secolo per il distretto produttivo della Byzacaena e soprattutto per l'area di Nabeul, verificato anche nelle stratigrafie coeve della città, ad esempio nell'area della piazza porticata e delle terme (Fioriello et al, 2013: 287-93). Sempre in analogia con la situazione riscontrata nell'area urbana, a partire dalla fine del IV secolo e soprattutto tra V e VI, molto articolato è anche il repertorio delle anfore vinarie orientali (Late Roman 1, 2, 8, 10, 12).

Ulteriori elementi sul quadro insediativo del periodo tardoantico vengono dalle nove case/ fattoria, caratterizzate da concentrazioni di frammenti ceramici con estensione compresa tra i 500 e i 2000 mq. La distanza regolare rispetto alle ville (entro $1 \mathrm{~km}$ di raggio) sembra suggerire che questi nuclei minori dipendono direttamente da una villa di riferimento. La villa sembra dunque assumere una precisa funzione di controllo nello sfruttamento di un territorio organizzato in una fitta trama insediativa, in accordo con quanto osservato per il comprensorio della Puglia centrale da studi recenti (Campese, 2013-2014: 396-403).

Il passaggio all'Alto Medioevo si svolge nel segno della persistenza insediativa, in quanto nei siti delle ville individuate si riscontra la formazione di villaggi rupestri, sui quali si è avuto un interesse più marcato negli studi precedenti (da ultima Bertelli, 2007).
Questi villaggi tornano a sfruttare, come in età protostorica, le cavità naturali caratteristiche del territorio e presentano un'articolata diversificazione funzionale degli spazi, in cui spiccano gli ambienti per la produzione olearia in frantoi ipogei e le chiese rupestri contraddistinte da una particolare cura decorativa, come nei casi di Lama D'Antico, in continuità con la villa di Masseria Calderisi, l'edificio di culto di Masseria San Francesco nel sito della villa di Masseria Carbonelli, nonché l'insediamento rupestre di Masseria Citrone in continuità con la villa di Masseria San Leonardo. La documentazione materiale permette di riconoscere, in gran parte di questi contesti, una continuità di frequentazione fino al XIII secolo.

Sempre a questo periodo rimandano le ultime attestazioni di frequentazione della città dove, in seguito all'abbandono di gran parte dell'area urbana tra la fine del VI e gli inizi del VII secolo, per l'estensione delle aree umide ormai non più irreggimentate, l'abitato si restringe con un villaggio ben organizzato entro i limiti dell'acropoli, difesi da una fortificazione bizantina eretta nella seconda metà del VI secolo lCampese, Caggese \& Cuccovillo, 2013), la cui sopravvivenza è attestata ancora nel XII, quando il geografo Guidone ricorda Egnazia come oppidum tra i boschi.

\section{Bibliografia}

Aprosio M, 2008: Archeologia dei paesaggi a Brindisi dalla romanizzazione al Medioevo, Edipuglia, Bari.

Bertelli G, 2007: Struttura e morfologia degli insediamenti rupestri, in Menestò E (ed.), Puglia tra grotte e borghi. Insediamenti rupestri e insediamenti urbani: persistenze e differenze, Atti II Convegno sulla civiltà rupestre, Savelletri di Fasano, 24-26 novembre 2005: 93-117. CISAM, Spoleto.

Biancofiore F, E Ghisellini, C Santoro, P Serafin Petrillo \& MV Fontana 1994-1995: Egnazia (Brindisi), scavi 1966 sull'acropoli di Egnazia, Notizie degli Scavi di Antichità, 11, 165-204.

Burgers G-J, 1998: Constructing Messapian Landascapes, Brill Academic, Amsterdam. 
Cambi F, 2001: Calabria romana. Paesaggi tardo repubblicani nel territorio brindisino, in Lo Cascio E \& Storchi Marino A (ed.), Modalità insediative e strutture agrarie nell'Italia meridionale in età romana: 363-89. Edipuglia, Bari.

Campese M, 2013-2014: Insediamenti urbani e rurali della Puglia centrale tra l'età tardoantica e la conquista normanna, tesi di dottorato di ricerca in Storia e Archeologia globale dei paesaggi, Università degli Studi di Foggia.

Campese M, M Caggese \& M Cuccovillo 2013: La fortificazione bizantina dell'acropoli di Egnazia, in Bartoloni G \& Michetti LM (ed.), Mura di legno, mura di terra, mura di pietra: fortificazioni nel Mediterraneo antico, Atti del Convegno internazionale, Roma, 7-9 maggio 2012: 242-45. Quasar, Roma.

Casavola L, 2002: Mola Paduano. Le anfore, in Ciancio A (ed.), La Peucezia in età romana: il quadro archeologico e topografico: 53-81. Progedit, Bari.

Cassano R, 2007: Egnazia al tempo della diocesi, in Carra RM (ed.), La cristianizzazione in Italia fra Tardoantico ed Altomedioevo: aspetti e problemi, Atti del IX Congresso Nazionale di Archeologia Cristiana, Agrigento, 20-25 novembre 2004: 1259-82. Saladino Editore, Palermo.

Cassano R, 2008-2009: Egnazia tardoantica: il vescovo protagonista della città, Rendiconti della Pontificia Accademia Romana di Archeologia, 81, 15-37.

Cassano R, 2009a: La vicenda urbana di Egnazia ridisegnata dalle recenti indagini, www.fastionline.org/ docs/FOLDER-it-2009-161.pdf

Cassano R, 2009b: L'area archeologica di Egnazia, in Cassano R \& Fioriello CS (ed.), Percorsi di storia. Itinerari informativi nel territorio di Fasano: 37-75. Gelsorosso, Bari.

Cassano R, 2011: Santuari di età repubblicana a Canosa ed Egnazia, in Bertoldi Lenoci L (ed.), Canosa. Ricerche storiche 2010, Atti del Convegno di Studio, Canosa, 12-13 febbraio 2010: 243-62. Edizioni Pugliesi, Martina Franca.

Cassano R, G Mastrocinque, A Mangiatordi \& CS Fioriello 2013: Urbanistica, economia e società ad Egnazia tra la fine del IV e il V secolo d.C., in Colangelo A, Pellacchia D \& Stigliano A (ed.), Potere e politica nell'età della famiglia teodosiana (395-455). I linguaggi dell'impero, le identità dei barbari: sintesi regionali, Atti del Convegno, Ravenna, 23-24 Settembre 2010: 267-81. Edipuglia, Bari.

Cassano R, V Di Grazia, CS Fioriello, A Pedone \& L Tedeschi 2004: Ricerche archeologiche nell'area del “foro" di Egnazia. Scavi 2001-2003: relazione preliminare, Epigrafia e territorio. Politica e società, Temi di antichità romane, 7, 7-98.

Cassano R, CS Fioriello, A Mangiatordi \& G Mastrocinque 2007: Indagini archeologiche nella città di Egnazia. Scavi 2004-2006: relazione preliminare, Epigrafia e territorio. Politica e società, Temi di antichità romane, 8, 7-136.

Chelotti M, 2007: Regio II, Apulia et Calabria. Gnatia, in Panciera S (ed.), Supplementa Italica, vol. 23: 467-86 Quasar, Roma.

Cocchiaro A, 1991: Fasano (Brindisi), Vuotano Piccolo, Taras, 11, 2, 275-76.

Cuccovillo M, 2010: Lo sfruttamento delle aree incolte, in Todisco L (ed.), Archeologia e Storia della Puglia centrale dall'età del Bronzo all'alto Medioevo, Atti del Convegno di Studi, Bari, 15-16 giugno 2009: 41519. L’Erma di Bretschneider, Roma.

D’Eredità A \& A Caringella 2011: Le anfore, in Bertelli G \& Lepore G, Masseria Seppannibale Grande in agro di Fasano (BR). Indagini in un sito rurale (a.a. 20032006): 452-68. Adda Editore, Bari.

Favia P \& R Giuliani (ed.) 2011: Gli edifici nella parte meridionale del sito, in Small A led.) Vagnari. Il villaggio, l'artigianato, la proprietà imperiale: 143-229. Edipuglia, Bari.

Fioriello CS (ed.) 2008: Rotte mediterranee della cultura. Turismo integrato e riuso delle architetture, Atti del Convegno Internazionale di Studi (Fasano, 18-19 settembre 2008), Gelsorosso, Bari.

Fioriello CS, C Annese, M Caggese, R Conte, G Disantarosa, M Foscolo, V Giannico \& D Palmisano 2013: Nuovi dati sulla circolazione delle merci a Egnazia tra IV e V secolo: importazioni e produzioni locali, in Colangelo A, Pellacchia D \& Stigliano A (ed.) 2013: Potere e politica nell'età della famiglia teodosiana (395-455). I linguaggi dell'impero, le identità dei barbari: sintesi regionali, Atti del Convegno, Ravenna, 23-24 Settembre 2010: 282-305. Edipuglia, Bari.

Giuliani R, 2010: Modificazioni dei quadri urbani e formazione di nuovi modelli di edilizia abitativa nelle città dell'Apulia tardoantica, in Volpe G \& Giuliani 
$\mathrm{R}$ (ed.), Paesaggi e insediamenti urbani in Italia meridionale fra Tardoantico e Altomedioevo, Atti del Il seminario sul Tardoantico e l'Altomedioevo in Italia meridionale, STAIM 2, Foggia-Monte Sant'Angelo, 27-28 maggio 2006: 129-66. Edipuglia, Bari.

Guida Egnazia, 2015: Guida al MArE - Museo Nazionale Archeologico di Egnazia 'Giuseppe Andreassi' Itesti di A. Cinquepalmi, R. Cassano, A. Cocchiaro, G. Mastrocinque), Quorum Edizioni, Bari.

Mangiatordi A \& M Campese 2008: Paesaggio rurale dell'Ignatinus ager: metodologie e prospettive di ricerca, in Fioriello CS (ed.), Rotte mediterranee della cultura. Turismo integrato e riuso delle architetture, Atti del Convegno Internazionale di Studi, Fasano, 18-19 settembre 2008: 115-42. Gelsorosso, Bari.

Mangiatordi A \& CS Fioriello 2013-2014: Egnazia in età romana: aspetti del paesaggio, Taras, 33-34, 125-42.

Mastrocinque G, 2008: Le terme del foro, Scheda di approfondimento, in Fioriello CS (ed.) 2008: Rotte mediterranee della cultura. Turismo integrato e riuso delle architetture, Atti del Convegno Internazionale di Studi, Fasano, 18-19 settembre 2008: 95-102. Gelsorosso, Bari.

Mastrocinque G, 2014: Spazio residenziale e spazio produttivo ad Egnazia in età tardoantica, in Pensabene P \& Sfameni C (ed.), La Villa restaurata e i nuovi studi sull'edilizia residenziale tardoantica, Atti del Convegno Internazionale del CISEM, Piazza Armerina, 7-10 novembre 2012: 415-26. Edipuglia, Bari.

Mastrocinque G, c.s.: Le terme del Foro, in Canano R, Mastrocinque G, Ricerche archeologiche nella città di Engnazia. Scavi 2007-2015, in Chelotti M, Silvestrini M (ed.), Epigrafia e Territorio. Politica e Società X. Edipuglia, Bari.

Semeraro G, 2009a: Castelluccio e il sistema insediativo dall'età del Ferro all'età arcaica, in Burgers GJ \& G Recchia (ed.), Ricognizioni archeologiche sull'altopiano delle Murge. La carta archeologica del territorio di Cisternino: 73-87. Claudio Grenzi Editore, Foggia.

Semeraro G, 2009b: Strumenti per l'analisi dei paesaggi archeologici. Il caso della Messapia ellenistica, in Osanna M (ed.), Verso la città. Forme insediative in Lucania e nel mondo italico fra IV e III sec. a.C., Atti delle Giornate di Studio, Venosa, 13-14 maggio 2006: 289-306. Osanna Edizioni, Venosa.
Silvestrini M, 2005: Le città della Puglia romana. Un profilo sociale, Edipuglia, Bari.

Small A (ed.) 2011: Vagnari. Il villaggio, l'artigianato, la proprietà imperiale, Edipuglia, Bari.

Stellati A, 2012-2013: Le dinamiche di gestione delle risorse vegetali ad Egnazia (BR) tra età romana e Medioevo: un approccio integrato, tesi di dottorato di ricerca in Storia antica, Archeologia classica, Diritto romano, Università degli Studi di Bari Aldo Moro. 